\title{
Conversaciones juveniles: aportes a las prácticas y lógicas de la investigación social
}

\author{
YOUTH TALKING: NOTES ON SOCIAL RESEARCH PRACTICES AND LOGICS
}

Klaudio Duarte (claudioduarte@u.uchile.cl) Departamento de Sociología, Universidad de Chile (Santiago, Chile) ORCID: 0000-0001-7855-4300

Manuel Canales (mcanalesc@gmail.com) Departamento de Sociología, Universidad de Chile (Santiago, Chile) ORCID: 0000-0002-5197-7642

Pablo Cottet (pcottet@yahoo.com) Departamento de Sociología, Universidad de Chile (Santiago, Chile) ORCID: 0000-0003-3431-3307

\begin{abstract}
Social research techniques are a set of devices that contribute to the observation and knowledge of the social. Such devices are the subject of analysis in this article. The main argument that we hold is that increasingly innovation in design and use is required to better understand the complexity contained in social processes we studied, not succumbing to the formalization and crystallization of the same, but opening to movements that blur boundaries and open up new possibilities increasingly filled with everyday observation. The youth as a metaphor for the social contains a good opportunity for the deployment of these ideas, especially in the possibility of addressing the confluence of biography and structure, when these individuals reflect on their future. With a qualitative method we investigate how young people from different social sectors are experiencing the output of secondary education in Chile. Further analysis on the technique used in this research (and decisions that demonstrate our logical and research practices) allows us to offer the following thoughts.
\end{abstract}

Key words: social research, techniques for the production of information, group interview, group discussion, class council.

\section{Resumen}

Las técnicas de investigación social constituyen un conjunto de dispositivos que contribuyen a la observación y conocimiento de lo social. Dichos dispositivos son el objeto de análisis del presente artículo. El argumento principal que sostenemos es que se requiere cada vez más innovación en su diseño y uso, para dar mejor cuenta de la complejidad contenida en los procesos sociales que estudiamos, no sucumbiendo ante la formalización y cristalización de las mismas, sino abriendo a movimientos que diluyan los límites y abran nuevas posibilidades cada vez más llenas de la cotidianidad del fenómeno en observación. Lo juvenil como metáfora de lo social, contiene una buena oportunidad para el despliegue de estas ideas, sobre todo en la posibilidad de provocar la confluencia de biografía y estructura, cuando estos sujetos reflexionan sobre su futuro. Con un método cualitativo indagamos sobre cómo jóvenes de 
diversos sectores sociales están experimentando la salida de la enseñanza media en Chile. Un análisis posterior sobre la técnica utilizada en dicha investigación, las decisiones que evidencian nuestras lógicas y prácticas investigativas, nos permite ofrecer las siguientes reflexiones.

Palabras clave: investigación social, técnicas para la producción de información, entrevista grupal, grupo de discusión, consejo de curso.

\section{Introducción}

El siguiente texto ubica como asunto de reflexión el modo en que el oficio sociológico se despliega en un proceso de investigación social, abordando específicamente la cuestión de las conversaciones como fuente nutritiva para ese conocimiento, así como se despliegan nuestras lógicas y prácticas en esos procesos. Las técnicas formalizadas en la literatura especializada bajo denominaciones del tipo "grupos de discusión", "grupos focales", "entrevistas grupales", "testimonios colectivos", han concurrido en una investigación social singular, sobre "lo juvenil", de manera híbrida y complementaria, expandiendo sus fronteras. Las implicancias y aprendizajes para el oficio investigativo, que dicha complementariedad y confluencia aportan a la promoción de conversaciones sociales, son el eje de este texto.

Estas conversaciones sociales juveniles, se desplegaron en el proceso de producción de información en la investigación desplegada en el marco del Proyecto Anillo "Juventudes: transformaciones socioeconómicas, sociopolíticas y socioculturales de las y los jóvenes en el Chile contemporáneo", que se implementó desde diciembre 2012 a mayo 2016, en un trabajo mancomunado de 15 investigadores e investigadoras de siete universidades y dos centros de estudio. Una de sus fases investigativas buscó abordar las subjetividades juveniles del Chile actual, a través de una comprensión / interpretación de la reproducción y análisis de sus relatos en referencia a un momento autobiográficamente relevante y de pertinencia general: el egreso de la enseñanza secundaria.

La globalidad de la investigación estuvo guiada por la idea de que las juventudes son una buena metáfora de la sociedad en que viven, en tanto ellas corporizan en sus biografías los procesos sociales de continuidades y cambio que van marcando las épocas y los relieves de los procesos históricos. En particular, asumimos que en dichas biografías existen diversos momentos que, a modo de pasos, van estableciendo marcas que condicionan los modos de ser y hacerse joven en cada época. Por lo cual, tomar algunos de esos pasos nos podía permitir la exploración de la condensación de los múltiples factores que evidencian la complejidad de lo social en esas experiencias, y que constituyen lo que modernamente se denomina lo juvenil.

En el presente texto desplegamos las coordenadas metodológicas con que se llevó a cabo el estudio. Primero profundizamos en algunas cuestiones sobre el objeto de nuestro estudio: las preguntas que guiaron la conversación social para acceder a él; posteriormente damos cuenta cómo se llevaron a cabo técnicamente las conversaciones para generar "los datos" de la investigación, comentando sus alcances y limitaciones. $Y$ en el ítem final, reflexionamos nuestros aprendizajes, en el orden epistémico y metodológico, en torno a las técnicas conversacionales y la conversación como oportunidades para el conocimiento de lo social, así como la necesidad de innovar en el diseño y uso de dichas técnicas. 


\section{Conversaciones juveniles sobre salir de "cuarto medio"}

\section{Nuestro objeto de análisis}

Para producir la información en la investigación global que ya señalamos, definimos el uso de una técnica conversacional grupal sobre "¿cómo están viviendo este paso?" las y los jóvenes estudiantes. El debate del equipo investigador transitó buscando descubrir si aquello a indagar se posaba, sobre la vertiente de las valoraciones e ideas circulantes -el deber y los ideales- que permite conocer el grupo de discusión, o si el eje indagatorio se instalaba más bien en cuestiones del orden vivencial-experiencial, en que el sentido de la acción juega un rol preponderante, a lo que se puede acceder más bien a través del grupo focal. Asumimos que en nuestra disciplina existen dos técnicas ya conocidas y con legitimidad en la comunidad científica: el grupo de discusión y los grupos focales, respectivamente. Se plantea que ambas, "ocupan un lugar a caballo entre los modos principales de obtención de información cualitativa en las ciencias sociales: las técnicas de entrevista individual y las técnicas de observación participación" (Valles 1999:294).

Para nuestro equipo investigador ambas vías parecían pertinentes y factibles para la indagación crítica de los modos en que la cuestión educacional configura subjetividades y estructuraciones que podían evidenciar la metáfora de los últimos cuarenta años en el Chile neoliberal. Se instaló en nosotros el debate sobre cuál de las dos vías era más pertinente para el propósito definido. Surgió entonces la interrogante sobre las posibilidades de mezclar, en el mismo ejercicio conversacional, ambas formas de provocar el habla, hasta antes siempre estandarizadas, enseñadas, transmitidas y legitimadas como separadas.

Esta curiosidad investigativa abrió un posible hallazgo no intencional del proceso investigativo, como muchos en la investigación social: la posibilidad de que propuestas como el multimétodo, en la mezcla polietápica de técnicas, pudiera agudizarse ahora en la mixtura, en la técnica misma, de más de un modo de hacer investigación. En nuestro caso singular se trató de provocar conversación juvenil, desclasificando los protocolos autorizados por la literatura metodológica de las ciencias sociales.

\section{Relaciones entre el objeto de este artículo y las preguntas de la investigación}

Nos planteamos que analizar estos momentos biográficos, es conocer las trayectorias subjetivas, observadas en esos lugares de tránsito donde cada sujeto se "detiene" separando una fase socializadora de otra. En esa detención momentánea, se instala un tiempo propio -el de estar haciendo el egreso, el salir de- donde la subjetividad aparece reflejada y reflexionada de modo instituyente, en sentido fuerte de lo autobiográfico.

Los sentidos del pasaje que buscamos escuchar tienen la peculiaridad de lo que antaño se refería a los ritos de pasaje, en que el dolor era la clave de comprensión de aquello que marcaba un pasado, así como la oportunidad un nuevo futuro para cada individuo y su grupalidad. Aquí los actualizamos como momentos cruciales: lo que cotidianamente es camino continuo, en tales momentos del pasaje de una fase a otra, acontece un cruce de opciones o cuando menos reclasificaciones sociales. El sujeto resulta así reflexionado sobre la estructura social y, al contar el sentido de sus acciones (actuales, futuras), lo mismo informa de su propio proyectarse, que de la formación socio-histórica en la que resulta proyectado, como "la realidad" vivida en la que se ve actuando en tanto sujeto.

Nuestra pregunta de investigación -el relato de esos dos tránsitos o trashumancias- logró articular de modo fuerte dos ejes: estructura social y autobiografía. En vez de liquidar el concepto de lo juvenil, por su 
contingencia epocal y estructural -lo joven varía histórica y estratificadamente-, optamos por integrar ambas condiciones sobre una tercera, la biografía, y más específicamente, la autobiografía de quienes viven y actúan en esas épocas y esas estructuras sociales.

Dichos momentos condensan biografía (pues tienen eficacia para identificar: "marcan"), estructura social (pues relacionan al sujeto con la red de puestos y caminos sociales) y eventualmente época (pues pueden relacionar inter e intra generacionalmente). Así, esta estructura social pudimos observarla expresada en las subjetividades a que da lugar, esto es, en los modos de significar y dotar de sentido a las trayectorias sociales que ofrece a las y los sujetos: "Desde esta perspectiva, el problema fundamental de la investigación en ciencias sociales sería comprender que el conocimiento constituye una forma de práctica social que no surge en el vacío y que debe ser analizado en un contexto que adquiere sentido desde un cúmulo de relaciones sedimentadas en un trasfondo de naturaleza semiótico-material. A partir de lo anterior, proponemos entender el conocimiento como una 'acción situada', es decir, como el producto de un trasfondo corporal e histórico en el cual se articulan saberes y prácticas, con artefactos, espacios y tradiciones que hacen parte de una forma de vida" (Sandoval 2013:38).

Cuando analizamos esos momentos estamos conociendo la estructura social en que el sujeto se observa actuando; esto es, la malla de posiciones sociales y la de caminos entre ellos, con sus correspondientes diferenciales de valoración. Aquello es la "realidad" o el "mundo" en que cada sujeto inscribe su propio actuar; el contexto donde toma sentido su hacer, como lo dado, lo posible, lo imposible, entre otras variantes.

Lo que puede revelarse entonces, en el relato de estos pasajes, es la modulación progresiva de la subjetividad -como un camino de formación del sujeto- cara a cara con la estructura social.

Este enfoque tiene una restricción importante. Observamos la subjetividad en el contexto de sus procesos institucionalizados. Así, observamos lo social produciendo jóvenes, y dejamos oscuro el campo de la autoproducción de lo juvenil, como de modo paradigmático se abre con los estudios de culturas juveniles. Esto es, observamos jóvenes en los caminos institucionales. Dos argumentos justifican, sin embargo, esta pérdida: a) los momentos son institucionales y fronterizos, pues indican un transitar entre dos instituciones: desde la educación media a la educación superior, desde la educación media al trabajo asalariado, o desde la educación media a un estado sin educación superior ni trabajo, en la versión no institucional. Entonces, lo institucional que sigue presente, se encuentra con la subjetividad activada por este pasaje, "detenida", reflexiva, algo poco común en las cotidianeidades institucionales; b) estudiar estos pasajes, y las trayectorias que las cuentan o anuncian, es observar a las formaciones sociales en su eficacia "ideológica", esto es, en su capacidad de ofrecer a los jóvenes puestos y caminos que hagan biografías sustentables, o en su defecto, de instalar cuadros de anomias o reflexión crítica.

También pasamos de tratar la cuestión de lo juvenil bajo las categorías canónicas de la transición y la moratoria: "Por eso aquí llamamos la atención sobre la necesidad de trabajar con una conceptualización no ontológica de las distinciones culturales al momento de analizar sus articulaciones materiales e históricas en los procesos de configuración identitaria. Para el caso de la clasificación social de la edad en las ideas de juventud, resulta imprescindible desmontar toda concepción de la diferencia etaria como homogénea en su interior. Se trata, más bien, de un espacio de despliegue y de disputa de distintos procesos de regulación ideológica que procuran fijar el sentido y las fronteras de admisibilidad social de ciertos grupos y perfiles «juveniles» en base a este criterio" (Elizalde 2015:139). 
Esta vez, buscamos en las inter-fases, el modelarse progresivo y acumulativo de la subjetividad juvenil. Nada de espera o demora: sino más bien intensa subjetivación del presente, pues, en el momento de los pasajes que aquí estudiamos, pasado y futuro se acoplaron en el ahora. Pero tampoco un presente encerrado sobre sí -como en el ocio y el socializar extra-institucional- sino en una trayectoria, andando cada pasaje.

De esta manera, las preguntas que orientaron nuestra búsqueda fueron: ¿qué imagen social se proyecta en esos relatos: cómo es el mundo, la realidad en que la acción/elección del sujeto encuentra el fondo para su "sentido"?, ¿qué subjetivación permiten esos relatos, en términos de su eficacia "ideológica"?

Y la provocación inicial con la que abrimos cada la conversación fue: ¿cómo están viviendo la experiencia de salir de cuarto medio?

\section{La operación conversacional}

\section{Sobre la técnica}

Como adelantamos en el proceso de construcción de nuestro objeto, nos planteamos provocar conversaciones que escaparan a las esquematizaciones clásicas de la técnica como "grupo de discusión", "grupo focal" o "entrevista grupal". Lo importante es que las conversaciones eran sostenidas por jóvenes de los mismos cursos escolares, en el momento mismo en que dicha sección visualiza también su disolución inminente. En ese sentido, las reuniones constituyeron un verdadero acontecimiento reflexivo: así por lo que está en juego, como por el contexto de enunciación.

A nuestro juicio, la forma de la conversación es armónica con su experiencia. El momento del egreso es al mismo tiempo individual y sobre todo individualizante respecto al grupo, y una vivencia grupal y comunitaria (no es infrecuente, como se sabe, la intensa comunidad que se gesta en la grupalidad escolar al acercarse el egreso).

Así, las conversaciones no fueron propiamente de "opinión", sino que se acercaron directamente al testimonio en grupo. Dicha escena, donde los y las jóvenes concurren testificando respecto de sus pasos, sus pasajes, ante ese grupo que les conoce y del que también ya empiezan a despedirse, les revela, también ante sí mismos, como la subjetividad que habitan buscándola y el mundo en el que se dicen sujetos.

Se trata de una técnica de investigación -en el sentido que este estudio la utilizó- pero fue también un evento comunitario -en el sentido que incita a las y los participantes a testificar su proyecto de sí. Finalmente constituye una ritualización del pasaje, tematizándolo y actuándolo en grupo.

\section{Sobre la guía de la conversación}

La conversación fue guiada por un investigador o investigadora, cuya tarea fue lograr que el grupo trajera a esa conversación todo el fondo discursivo con el que los sujetos/as pueden entender y comprender el objeto de la investigación: sus subjetividades y sus circunstancias, en los pasajes indicados. Por ello no utilizamos una pauta de preguntas, sino más bien, cada facilitador/a se dispuso como un posibilitador del habla, alimentándola con preguntas internas o plausibles de hacer en el contexto de la conversación, así como llevando las reflexiones a los límites que el grupo pueda recorrer. 
No se trata, como es obvio, de una conversación abstracta ni analítica. Convocamos a las y los jóvenes a reflexionar sus propios pasajes presentes y reconocerse en sus imaginarios y proyectos. La imaginamos como una conversación abierta sobre el futuro hacia el que se proyectan y el presente en que imaginan esa proyección. Para efectos simplemente de apoyo, en caso de una conversación trabada, dispusimos de recursos básicos como pedir comparación entre el presente y el pasado o entre hombres y mujeres o entre clases sociales, etc. Sin olvidar que no se trataba de reemplazar los planteamientos propios de las y los jóvenes con una paleta exhaustiva de distinciones genéricas o etic. Pero la experiencia nos muestra que a veces algo de etic puede ayudar cuando lo emic trastabilla.

Lo que logramos generar es una discusión y al mismo tiempo un intercambio de testimonios. La discusión y el foco van articulándose constituyendo grupo por autonomía conversacional y por la intervención de quien facilita para provocar profundidad.

El tema es existencialmente desafiante/desafiado, y por ello pudo provocar su decir como sujetos, que es en última instancia la función de la técnica con que, quien investiga, sondea o ausculta su objeto de modo informativo. Por lo mismo, quien moderó estuvo preparado para situaciones complejas, según sea crítica la cuestión que analizamos. En un colectivo, por ejemplo, de jóvenes de Liceos Municipales de sectores empobrecidos, encontramos que el tema gatilla habla testimonial y catártica. En ese sentido, nos correspondió contener la conversación e invitar a hacer un análisis de las causas compartidas de aquello así, se saca al hablante de su subjetivación de la crisis de integración social, y le lleva a hacer la crítica política y social de aquella. Hasta puede ser, entonces, que la catarsis acelere también algún proceso de toma de conciencia no ingenua ni pasiva de su situación. ¿Qué otra cosa puede hacer quien investiga?

\section{Sobre el análisis}

En esta mixtura -de complementariedad y confluencias- nos planteamos que el análisis de la información producida debía lograr comprender las subjetividades juveniles ahí planteadas y entenderlas en el fondo de una formación histórico-social, sus estructuras y sus procesos.

Por lo mismo, escuchamos los relatos tratando de identificar los patrones o pautas que los organizan y luego, en otra cara, los interpretamos desde una teorización sobre la estructura social (por qué ese discurso desde esa posición en la estructura) y el proceso social (porque ese discurso en la actualidad, sus pasados y sus futuros). La confluencia de ambos, estructura y proceso, dan buena cuenta de lo social chileno contemporáneo que, como en una metáfora, las y los jóvenes evidencian.

\section{Aprendizajes para el debate}

En esta sección, a modo de aprendizajes, quisiéramos compartir algunas comprensiones, o líneas de pensamiento, que nos provoca el hecho de haber innovado, desde las técnicas de conversación grupal, hacia un dispositivo del tipo "consejo de curso auto-autorizado" (en el sistema escolar chileno, se cuenta con una actividad programada semanalmente con horario fijo denominada "consejo de curso", en la que "el/la profesor/a jefe" coordina el tratamiento de temas de interés común a la vida cotidiana del curso).

Para ello partimos de una propuesta según la cual las técnicas de investigación social son dispositivos que provocan el habla (encuestas, entrevistas, grupos focales, grupos de discusión, observación participante, relatos/historias de vida). Los datos corresponden a específicos modos de decir, obtenidos mediante la aplicación de determinados juegos de lenguaje. 
Ello funciona para el cometido de aproximarse a conocer lo social, porque lo que investigamos se constituye en y por el decir. Como se ha señalado: "lo social es del orden del decir" (Alvira, Ibáñez y García 2005:11). La mentada confluencia de estructuras y procesos tiene lugar en ese orden del decir, también y no únicamente. Este orden es un régimen propio a la acción, concepto que está en el crisol histórico de lo social. Olvidar el central sitio del concepto de acción en los debates sobre lo social, termina convirtiéndolo en una sustancia, sustantivándose en "cosas" y/o "humanos".

Reparemos en el inaugural papel del metaforizar en la constitución moderna de lo social: metáfora de lo común y corriente. No inventamos nosotros que los y las jóvenes constituyen una posición de sujeto que metaforiza lo social, al menos no del todo. Lo han venido haciendo tanto los y las jóvenes de las formaciones sociales históricas modernas y contemporáneas, en su condición de interrogadores e interrogados, como también las ciencias sociales que los han estudiado como una interrogación científica, aplanada, puesta bajo la pregunta por y desde el orden, por y desde mathema. Tanto lo que estudia la ciencia, como la propia ciencia; en este caso tanto los y las jóvenes, como las ciencias sociales de lo juvenil: metaforizan lo social.

Las y los jóvenes metaforizan lo social interrogado y como interrogación en un devenir minoritario que incomoda e insiste, que no termina de calzar con las representaciones del orden adultocéntrico. Las ciencias sociales de lo juvenil no terminan de elaborar el mandato moderno de conocer ordenando, para ordenar lo que se conoce. Este, nos parece, es el régimen epistemológico de las metodologías y técnicas de investigación en ciencias sociales que, lo juvenil como metáfora, nos retroalimenta para interrogar las lógicas y prácticas de la investigación social contemporáneas, contexto de la innovación técnica experimentada en el estudio que aquí informamos.

Especialmente reveladora de la metaforización de lo social es la conversación juvenil cuando trata de su egreso de la enseñanza media (secundaria), ya que queda situada en el futuro, en los futuros. Se trata de un analizador poderoso sobre las expectativas biográfico-generacionales, de cada quien inscrito en las redes e instituciones. Hablar del futuro entre jóvenes que están en su último año de enseñanza secundaria (cuarto medio) en Chile, es sin más hablar del futuro en general, hablar de la vida, la biografía, el sentido de los caminos que se visualizan y sus destinos.

Se habla por la herida. Por eso se buscó el mejor modo y dinámica para proponer tal conversación, así resulta un dispositivo basado en una conversación grupal, sobre su posición/movimiento estructural (egresar de la enseñanza secundaria), e invita a hablar sobre tal experiencia, tal posición/movimiento en la estructura social, en momentos cruciales o de pasaje. Para lograrlo, el dispositivo técnico utilizado resultó uno de ciertas modificaciones adaptativas de la práctica "consejo de curso". Las modificaciones esenciales fueron:

a) Realizándose en la sala de clases, no cuenta con la presencia del/la profesor/a jefe, de modo que la reunión desconecta algo de su lazo explícito y jerárquico con la institucionalidad a la que pertenece en su forma de práctica cotidiana. Así, el consejo de curso se desplaza a una frontera -la de la paridad total- aun sin llegar completamente a ella, pues sigue aconteciendo en contexto institucional y ante un adulto, el/la investigador/a.

b) La conversación no se orienta hacia decisiones, o acuerdos, como en el regular consejo de curso, sino al juego del habla opinante, y en este caso, testimonial. No es un consejo de curso que decide, sino a lo más uno que aconseja. Así, la forma "pragmática" de la práctica cede su lugar a una nueva forma suavizada 
de ser grupo cuya práctica sea la de la escucha: la forma más propia de grupalidad que elabora y articula significados comunes a experiencias comunes (grupalidad "semántica" y "sintáctica").

c) De este modo, la transformación de la práctica permite el desarrollo de su cara más propiamente grupalista -la grupalidad de la escucha-, del acompañamiento, del nosotros grupo básico, o del grupo en su cara de otro juez propio, menos la forma de grupo-equipo, como tiende, o debiera a tenderse en su forma más típica en el consejo de curso.

d) Se habla, como se ha dicho, de un asunto crucial biográfica, colectivamente e institucionalmente: el egreso, la partida o separación, entre ellos, y de ellos con la institución aun "protectora". Al egresar, "vamos en fila". Así, es una segunda frontera que, si no se traspasa completamente, se bordea al hablar anticipando la separación, esto es, la distancia, aun cuando lo sea en esa intensa cercanía propia de este fin de ciclo.

Técnicamente el dispositivo funciona como una auto-entrevista en grupo, asistida por un promotor, quien mientras menos intervenga más potencia la forma auto-entrevista grupal (que convoca a comparecer ante el egreso, focalizada en el egresar). Pero ambas modalidades son matices de lo mismo: una combinación de la dimensión grupal clásica de los grupos de discusión (orientación al consenso, esta vez como modo de grupo-escucha del momento que vive) y la propia de las entrevistas focalizadas, cuando tratan de temas cruciales (donde quien habla se deja ver, o entrevistar, de modo dilemático y simbólicamente cargado).

Para las lógicas y prácticas de la investigación social, modernas y contemporáneas, el vector técnico constituye una pieza aparentemente auxiliar, sin embargo decisiva, porque trafica códigos basales y silenciosos (o clandestinos) de los modos de conocer por parte de las investigaciones sociales. En la respuesta a la pregunta "¿qué técnicas pueden utilizarse para investigar científicamente tal realidad social?", hay ya un sentido común profesional cristalizado que muta secretamente -acríticamente- de recomendación en recomendación "especializada en metodología". Las técnicas (herramientas, instrumentos, etc.) objetivan lo que tratan (para conocerlo, apropiarlo y usarlo), lo simplifican (reducen objetivando) para luego adecuar lo tratado al concepto (abstracción) y pasarlas por totalidades sociales (generalizarlas).

Las técnicas de investigación social buscan adaptarse a formas históricas de metaforizar lo social. Así las encuestas son adaptaciones de formas censitarias de crear socius (votaciones, elecciones de decisiones individuales orientadas a decisiones colectivas); los grupos de discusión y focales, adaptaciones de formas colectivas de crear socius (asociaciones conversantes produciendo lo común); las entrevistas, adaptaciones de biografías inscritas en redes comunes. Es lo que se ha hecho en esta investigación: adaptar unas técnicas (grupos de discusión, entrevistas abiertas) a una forma social propia al sujeto estudiado (el consejo de curso).

En coherencia con Sandoval: "Desde esta perspectiva, el desafío metodológico es desarrollar modificaciones parciales a los dispositivos puramente conversacionales, con tal de escapar de la tendencia de los métodos cualitativos por saturar la pura dimensión semántica del lenguaje, sea a través de técnicas centradas en su componente referencial o anafórico (Ibáñez). Para ello, debemos implementar dispositivos activos de investigación -podríamos decir incluso, dispositivos dramatúrgicos- que permitan poner en acción lo que hay de fuerza performativa en la situación. La idea es no solo decir sino hacer con el lenguaje, sobrepasando los propios marcos a partir de los cuales se genera la reflexión: hablamos de investigar un actor actuando, analizar una PERFOMANCE en plena escenificación" (Sandoval 2013:42). 
El uso como dispositivo de investigación social de la conversación del curso modificada, realizada en el momento del egreso, puede ser pensado como el gesto técnico de adaptabilidad de las lógicas y prácticas de investigación social por homología: si el salir de cuarto medio es un rito de pasaje, el sujeto habla en propiedad si lo relata ante los suyos propiamente tales. Y pocos grupos competirán con la grupalidad del curso.

Pensamos que "los datos" que producen las técnicas de investigación social nunca son lo social, más bien forman parte de lo social, haciendo que la acción social, la vida social, se expanda y complejice, junto a otras innumerables prácticas y lógicas sociales. Esas mismas técnicas mutan sin coincidir nunca con lo social que objetivan, porque la vida social no es un objeto, no obstante, exige objetivarse sin fin, sin lograr "copiarlo" ni "capturarlo" del todo. La acción del orden del decir incorpora la realidad de los procesos de investigación, que operan metaforizando también como otras metaforizaciones históricas así lo juvenil.

Otra vez inventamos en esta experiencia investigativa de lo social: técnicamente promovimos la conversación entre jóvenes articulando condiciones testimoniales, opinantes e informativas. Ni entrevista grupal, ni grupo de discusión, tampoco testimonios grupales, de todas esas formas técnicas, extrajimos lo que permitía al sujeto joven, en situación de pasaje vital, decir su experiencia, para leer en ese decir lo social (es del orden del decir): biografía y estructura.

Otra vez inventamos, no fuimos los primeros y ni seremos los últimos: es la genealogía y arqueología de las ciencias modernas, también las sociales. No seremos nosotros quienes agreguemos una "nueva técnica" a las disponibles para investigar lo social. Nos parece mejor ofrecer esta perspectiva abierta que pudiese animar a atreverse a buscar esas sintonías entre las lógicas y prácticas de investigación social y un socius situado engendrándose ensamblado a otras prácticas y lógicas sociales.

Para evitar su cristalización es que eludimos nominarla, más bien insistimos en abrir objetivando. Al menos eso aprendimos y podemos compartir.

\section{Agradecimiento}

Artículo elaborado como resultado de la investigación del Proyecto Anillo JUVENTUDES financiado por CONICYT (SOC 1108). 


\section{Bibliografía}

Alvira, F; Ibáñez, J. y García-Ferrando, M. 2005. El análisis de la realidad social. Madrid: Alianza.

Elizalde, S. 2015. Estudios de juventud en el cono sur: epistemologías que persisten, desaprendizajes pendientes y compromiso intelectual. Una reflexión en clave de género. Última década 23(42): 129-145. doi: 10.4067/S0718-22362015000100007

Sandoval, J. 2013. Una perspectiva situada de la investigación cualitativa en ciencias sociales. CINTA MOEBIO 46: 37-46. doi: 10.4067/S0717-554X2013000100004

Valles, M. 1999. Técnicas cualitativas de investigación social. Reflexión metodológica y práctica profesional. Madrid: Editorial Síntesis.

Recibido el 21 Jul 2016

Aceptado el 15 Sep 2016 